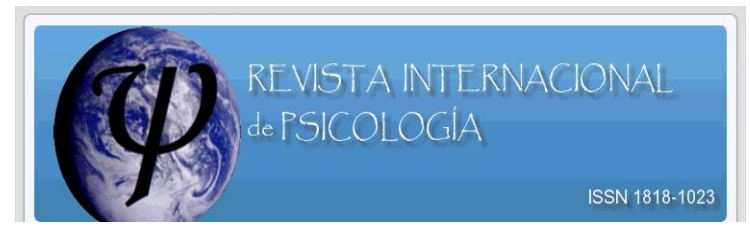

\title{
PREVALENCIA DE SÍNTOMAS DE ALTERACIONES MENTALES EN LA POBLACIÓN DE EL SALVADOR
}

\author{
José Ricardo Gutiérrez PS. MS. PhD ${ }^{1}$
}

\section{RESUMEN}

El presente estudio surge con la intencionalidad de describir y explicar algunas conductas consideradas como anormales, que presentan los salvadoreños y que en muchas ocasiones son el resultado de conflictos mayores. Como ejemplos, se tienen: La violencia social, violencia familiar. La delincuencia, homicidios, la depresión y ansiedad, entre otras. Los objetivos específicos que se plantearon al inicio del proyecto fueron: Identificar la incidencia de síntomas de trastornos mentales; Establecer la diferencia de síntomas en relación al sexo de la población; Determinar si el sexo incide en los trastornos mentales; y Establecer la prevalencia de síntomas de los trastornos mentales en cada zona geográfica del país. En la metodología del estudio, se utilizaron las estrategias siguientes: Se determinó que es un estudio descriptivo, su diseño de investigación es transacional descriptivo, Se utilizó el muestreo aleatorio por conglomerados, su técnica; la encuesta dirigida a 1,668 personas distribuidas en las 31 ciudad más importantes de El Salvador; como instrumento se utilizó el Cuestionario Ilustrado de Síntomas,(diseñado para la OPS/OMS) que mide la presencia de síntomas de diez trastornos mentales y del comportamiento. La discusión de los resultados se hace en la parte final de ésta reseña.

\section{Palabras Claves:}

Trastorno mental, salud mental, síntomas, Síndrome

\footnotetext{
${ }^{1}$ Universidad Tecnológica de El Salvador Correo electrónico: jrguty@yahoo.com
} 


\section{SUMMARY.}

The present study arises with the purpose of describing and explaining some behaviors considered abnormal, that Salvadorans present, which in many occasions are the result of greater conflicts; for example, social violence, familiar violence, delinquency, homicides, depression and anxiety, among others. The specific objectives that were considered at the beginning of the project were to identify the incidence of symptoms of mental upheavals; to establish the difference of symptoms in relation to the sex of the population; to determine if sex affects the mental upheavals; and to establish the prevalence of symptoms of the mental upheavals in each geographic zone of the country. In the methodology of the study, the following strategies were used: It was determined that it was a descriptive study, the design of investigation was transactional descriptive; the random sampling by conglomerates was used; the technique used was the survey directed to 1.668 people distributed in the 31 more important cities of El Salvador; the instrument used was the Illustrated Questionnaire of Symptoms (designed by the PAHO/WHO) that measures the presence of symptoms of ten mental upheavals and behavior. The results can be synthesized in percentages of prevalence of symptoms and some of these are the following: $50 \%$ of the interviewed people presented symptoms of compulsive obsessive upheaval, $47,7 \%$ presented anxiety symptoms and distresses, 36,8\% presented somatization characteristics. Also, 29,1\% presented some symptoms of depression; $33,2 \%$ of the evaluated population declared to have symptoms of the convulsive Syndrome. $35,8 \%$ demonstrated some suspicions of symptoms of an organic cerebral Syndrome. The final part of the study contains the conclusions and a set of solution strategies.

\section{Key word:}

Mental upheaval, mental health, symptoms, sindrome 


\section{INTRODUCCIÓN.}

En el estudio se describen y explican algunas conductas consideradas como anormales, que son evidenciadas cotidianamente en los salvadoreños y que en muchas ocasiones son el resultado o causas de conflictos mayores, tales como: La crisis delincuencial, la violencia familiar, crisis económica y problemas de personalidad; en éstos últimos, se pueden mencionar: La depresión, ansiedad y las neurosis. entre otras.

Los trastornos mentales, existen en el dominio de los fenómenos subjetivos; en el lenguaje que los constituyen, nada existe como verdad ontológica, ya que los fenómenos psíquicos no son cosas susceptibles de palpar y manipular objetivamente. La definición del trastorno mental o trastorno psiquiátrico; según el DSM-IV, es un "Síndrome o patrón comportamental o psicológico de significación clínica, que aparece asociado a un malestar, a una discapacidad o a un riesgo significativamente aumentado de morir, o de sufrir dolor, discapacidad o pérdida de libertad". Entre los paradigmas que explican el origen y las características de las alteraciones mentales, se tienen: La perspectiva socioeconómica de la salud mental, el enfoque de anormalidad, los modelos: médico, conductual, cognoscitivo y familiar sistémico y el aprendizaje por observación. Entonces, la salud mental es el bienestar psíquico y emocional del individuo; el cual se ve reflejado en la capacidad para enfrentar adecuadamente las exigencia de su vida, es decir, ser capaz de adaptarse al trabajo, a la familia y al entorno social en que se desarrolla.

\section{DISCUSIÓN Y ANÁLISIS DE RESULTADOS.}

El estudio, se realizó en una población, tanto del sexo femenino como masculino. tratándose que la muestra fuera distribuida proporcionalmente en relación al sexo de la población salvadoreña. Los resultados indican que cerca de dos tercera parte de la población estudiada, poseen un hogar establecido formalmente, descartándose la posibilidad que los síntomas existentes sean el resultado del divorcio, viudez o soltería de los salvadoreños, como lo plantean algunos autores. 
Este informe sugiere que cerca de la mitad de los entrevistados adolecen de dolores de cabeza; siendo éste un síntoma que caracteriza o indica la posibilidad de un trastorno mental. También puede ser indicador de una enfermedad de carácter orgánica. es decir, que las cefaleas, son un síntoma general que ésta presente en múltiples alteraciones a la salud. Por otra parte, más de la mitad de los evaluados se sienten nerviosos, tensos y aburridos; éstos síntomas están relacionados con la presencia de trastornos de obsesividad, ansiedad, somatización y neurosis, no se pudo determinar concretamente a que tipo de trastorno obedecen. Cuando se evaluó la capacidad para pensar bien, se encontró que más de la mitad de los encuestados, presentan confusión en sus pensamientos. Siendo éste un indicador importante vinculado con los trastornos de angustia, depresión, neurosis, psicosis y los síndromes cerebrales orgánicos; indicando que es un síntoma que puede estar presente en varios tipos de alteraciones mentales.

El estudio refleja que cerca de una quinta parte de los salvadoreños, han presentado la intención de acabar con su vida, es decir, que una segmento significativo han tenido la intención de suicidarse; siendo éste un síntoma claro y preciso de depresión. De igual forma, cerca de la mitad de la población, se sienten cansados; reflejando la existencia de síntomas de los trastornos de somatización, depresión y neurosis. Es importante destacar que las causas de los síntomas antes mencionados, no se pueden reducir a una sola condición o factor desencadenante, ya que éstos trastornos en su mayoría, son producidos por las condiciones sociales y ambientales donde convive el ser humano; como ejemplos se pueden citar: conflictos familiares, carencia de recursos, estrés laboral y conflictos emocionales.

Por otra parte, casi la mitad de la población encuestada, presentan la sensación de que alguien ha tratado de herirlos; lo anterior es un indicador o síntoma que puede estar presente en cualquiera de los trastornos de: obsesividad, paranoidismo y psicosis. También más de la mitad de los evaluados, se consideran más importantes de lo que piensan los demás; éste síntomas puede estar presente en los trastornos de paranoidismo, neurosis y psicosis. Asimismo, un segmento significativo de la población considerá que ha estado bebiendo o consumiendo drogas demasiado; indicador importante que refiere la presencia de alcoholismo 
y drogadicción como enfermedades que está incidiendo negativamente en la estabilidad psicológica, social, y orgánica de las personas.

En otra parte del estudio, se encontró que cinco de cada diez salvadoreños presentan síntomas, como: pensamientos repetitivos, ideas, imágenes, e impulsos que irrumpen una y otra vez en la actividad mental de las personas de forma estereotipada, los cuales son percibidos como desagradables e involuntarios; es decir, presentan la sensación de aflicción, inseguridad, angustia, miedo y que en la mayoría de veces surge sin explicaciones reales sobre sus causas; éstos síntomas son propios de los trastornos obsesivos compulsivos. Es de mencionar que los rasgos obsesivos en muchas ocasiones responden a trastornos neuróticos y paranoides. También se puede mencionar que de acuerdo a los expertos en salud mental, existe una correlación significativa entre los pensamientos obsesivos antes descritos y la depresión, que su prevalencia o frecuencia es igual en hombres y mujeres.

Es importante destacar que cerca de la mitad de la población estudiada presenta síntomas o características del trastorno de ansiedad y angustia; el cual se evidencia por la presencia de síntomas de aprensión, miedo pavoroso o terror, acompañado de sensación de muerte inminente. En momentos de crisis de la persona, aparecen síntomas como: falta de aliento, palpitaciones, opresión, malestar toráxico, sensación de atragantamiento o afixia y miedo a volverse loco y perder el control de su personalidad. Es de destacar que el trastorno de ansiedad y las crisis de angustia están vinculados con las características obsesivas compulsivas antes mencionadas, con los síntomas de depresión y con los rasgos neuróticos. Asimismo, más de una cuarta parte de la población, presenta síntomas de depresión, los que se caracterizan por perdida de capacidad para interesarse, motivarse y disfrutar de las cosas, disminución de su vitalidad y un cansancio exagerado que aparece después de un esfuerzo mínimo. También presentan disminución de la atención, falta de concentración, perdida de confianza en si mismo y la prevalencia de sentimientos de inferioridad,

Al continuar con la discusión de los resultados nos encontramos que cerca de cinco de cada diez de la personas estudiadas, presenta uno o más síntomas de alteraciones psicoticas, indicando que existe la posibilidad de una desorganización en la personalidad, distorsión de 
las percepciones, del pensamiento y las emociones, que pueden estar generando alteraciones de la concepción de la realidad. Es de recalcar que si la situación permanece por mucho tiempo, sin recibir ayuda o tratamiento, estas personas pueden presentar en un futuro a medianos plazo, déficit cognoscitivos y desencadenar alteraciones muy evidentes. Por otra parte, destacar que existen psicosis funcionales y orgánicas; en el presente estudio no se logro determinar si los síntomas antes descritos pertenecen a la primera o la segunda psicosis.

El informe indica que existe una clara diferencia en relación a la prevalencia de síntomas entre hombres y mujeres, ya que en la mayoría de las alteraciones, la presencia de síntomas en las mujeres es mucho mayor en comparación con la prevalancia en hombres. Al obtener un promedio de las diferencias, se encontró que existe un $7.2 \%$ mayor de síntomas en las mujeres. Sin embargo, es de destacar que se presenta una excepción, y es en la prevalencia de alcoholismo / drogadicción, donde los hombres superan a las mujeres en un 6.3\%. Estos resultados están en una estrecha relación con los planteamientos realizados por algunos especialistas en el sentido de que la mujer es más susceptible a padecer de algunos trastornos mentales y que éstos son precipitados por factores socioculturales, psicológicos y biológicos.

También en el análisis general de las incidencias de síntomas de algunos de los trastornos estudiados, se refleja que existe una prevalencia significativa; es decir, cuatro de cada diez de las personas evaluadas, presentan uno o varios síntomas de diferentes trastornos. Éste dato surge de la comparación que se hizo entre los diferentes trastornos estudiados; configurándose de esta manera, una tendencia al cuadro clínico neurótico-depresivo, con presencia de características psicoticas.

Por otra parte, si se comparan las estadísticas de la prevalencia de síntomas de trastornos mentales y del comportamiento, entre El Salvador y la prevalencia en otros países, se puede observar una mayor incidencia de síntomas en El Salvador; es decir, que en éste estudio se observa una prevalencia más significativa que en otros países. Sin embargo, los resultados coinciden perfectamente bien con los hallazgos de estudios realizados en países como: Chile, Brasil y Francia. Es oportuno destacar que en nuestros estudios no estamos hablando de 
diagnósticos definitivos, sino de prevalencia de síntomas; probablemente en los otros países se hayan considerado figuras diagnósticas más definitivas.

Finalmente queremos informar a nuestros amigos lectores que estén interesados en conocer el informe completo del presente estudio lo pueden encontrar en las bibliotecas y página web de la Universidad Tecnológica de El salvador.(htp/www.utec@edu.sv)

\section{REFERENCIAS}

Asociación Americana de Psiquiatría (2000) DMS-IV-TR, Editorial Masson, S.A. Barcelona, España.

ACISAM, (2001) Manual de intervención en crisis en caso de desastres, San Salvador, El Salvador.

Badía M.(2001) La salud de América Latina, Revista Entorno, editorial, Tecnoimpresos, San Salvador, El Salvador.

Bonilla G.( 2000 ) Estadística II, Métodos prácticos de inferencia estadista, UCA, editores, San salvador, El Salvador.

Bernal T. Metodología de la investigación, para administración y economía, editorial Printice hall, Colombia 2000.

Colegio Medico de El Salvador (1999 ) Propuesta Ciudadana por la Salud, Editorial, Tipografía Offset Laser, San Salvador, El Salvador.

Dempsey D, Zimbardo P. (1991), La Psicología y usted, Editorial Trillas, S.A. México.

Gutiérrez Q.( 2003) La familia salvadoreña, víctima de la exclusión, Revista Entorno, Editorial Tecnoimpresos, San Salvador, El Salvador.

Hernández S, Fernández C. Batista L, Metodología de la investigaron, editorial Mc Graw hill, México 2002.

Levav I (1992) Temas de salud mental en la comunidad, Serie Paltex, Organización Panamericana de la Salud. OPS.

Organización Mundial de la Salud, OMS, Informe de Salud en el Mundo 2001. 
Organización Mundial de la Salud, OMS (1995), CIE-10, Trastornos Mentales y del Comportamiento, editorial, Meditor Madrid España.

Sandler J, R.S. Davidson (1980), Psicopatología, editorial Trillas.S.A. México. Sue D, Et al (1999) Comportamiento Anormal, 4ª Edición, editorial Mc Graw Hill, México.

Vidal G. Alarcón,(1989) Psiquiatría, Editorial medica, Panamericana, Buenos aires, Argentina.

Zax M. Cowen E. L.(1985) Psicopatología, 2a . Edición, Editorial Interamericana, México.

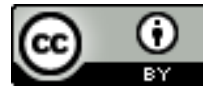

Este texto está protegido por una licencia Creative Commons 4.0.

Usted es libre para Compartir — copiar y redistribuir el material en cualquier medio o formato - y Adaptar el documen- to -remezclar, transformar y crear a partir del material- para cualquier propósito, incluso comercialmente, siempre que cumpla la condición de:

Atribución: Usted debe reconocer el crédito de una obra de manera adecuada, proporcionar un enlace a la licencia, e in- dicar si se han realizado cambios. Puede hacerlo en cualquier forma razonable, pero no de forma tal que sugiera que tie- ne el apoyo del licenciante o lo recibe por el uso que hace. 